\title{
BMJ Open Effect of basic public health service project on neonatal health services and neonatal mortality in China: a longitudinal time-series study
}

Pengyu Zhao, ${ }^{1}$ Xueyan Han, ${ }^{2}$ Lili You, ${ }^{2}$ Yu Zhao, ${ }^{3}$ Li Yang, ${ }^{2}$ Yuanli Liu (i) ${ }^{4}$

To cite: Zhao P, Han X, You L, et al. Effect of basic public health service project on neonatal health services and neonatal mortality in China: a longitudinal timeseries study. BMJ Open 2020;10:e034427. doi:10.1136/ bmjopen-2019-034427

- Prepublication history and additional material for this paper are available online. To view these files, please visit the journal online (http://dx.doi org/10.1136/bmjopen-2019034427).

Received 20 September 2019 Revised 23 April 2020 Accepted 30 May 2020

Check for updates

(C) Author(s) (or their employer(s)) 2020. Re-use permitted under CC BY-NC. No commercial re-use. See rights and permissions. Published by BMJ.

${ }^{1}$ Perking Union Medical College Hospital, Chinese Academy of Medical Science\& Perking Union Medical College, Beijing, China

${ }^{2}$ School of Public Health,

Chinese Academy of Medical

Science \& Peking Union Medical

College, Beijing, China

${ }^{3}$ Nursing Department, Peking

University First Hospital, Beijing,

China

${ }^{4}$ School of Public Health,

Peking Union Medical College

\& Chinese Academy of Medical

Sciences, Beijing, China

Correspondence to

Professor Yuanli Liu;

liuyl_fpo@126.com

\section{ABSTRACT}

Objective To analyse the trend change and level change of neonatal health services and neonatal mortality before and after the introduction of the Basic Public Health Service (BPHS) project in 2009.

Design and setting A national longitudinal study on neonatal mortality from 1991 to 2017 and neonatal health services from 2000 to 2017 was conducted based on data extracted from the National Neonatal Mortality Surveillance System and National Health Statistic Yearbook. The segmented linear regression model was used to assess the level changes and trend changes of the outcome variables before and after the introduction of BPHS project. Pearson correlation analysis as conducted to measure association between neonatal mortality rates (NMRs) and maternal health management rates (MMRs), neonatal visit rates, respectively.

Primary and secondary outcome measures The outcome variables were national NMR, the gap of NMR between urban and rural areas, maternal health management rate and neonatal visit rate.

Results The annual trend change coefficient of national NMR and the gap of NMR between urban and rural areas were $-0.57(p<0.01)$ and $-0.49(p<0.01)$ after the introduction of BPHS project, while the annual trend coefficient of the MMR and the neonatal visit rate were $1.21(p<0.01)$ and $0.85(p<0.01)$, respectively. The negative correlations were found between NMR and MMR $(r=-0.79, p<0.01)$ and neonatal visit rate $(r=-0.76$, $\mathrm{p}<0.01)$.

Conclusion The BPHS project was found to be associated with increased volume of neonatal health services and reduced NMR. The design and implementation of this project may provide references to other low-income and middle-income countries.

\section{INTRODUCTION}

Mortality at younger age is a key measure of population health. ${ }^{1}$ Reducing mortality rates among children under 5 years was one of the eight millennium development goals (MDGs). ${ }^{2}$ In the Sustainable development goals, reducing neonatal and under-5 mortality was also a priority. ${ }^{3}$ It had been reported that neonatal deaths account for $44 \%$ of the childhood mortality.
Strengths and limitations of this study

- This study focused on the neonatal mortality and neonatal health service in China before and after the introduction of the Basic Public Health Service (BPHS) in 2009.

- The neonatal mortality rates (1991-2017), maternal health management rates and neonatal visit rates (2000-2017) were analysed using the segmented linear regression model.

- The segmented linear regression model controlled auto-correlated errors and potential serial correlations in the longitudinal data while provided level change and trend changes of the outcome measures before and after the BPHS implementation.

- Due to limited data accessibility, this study did not assess the effectiveness of the BPHS project at the provincial or county levels.

- We could not control for other factors affecting the neonatal health outcomes through trend analysis, such as the health service quality, delivery place and so on.

Approximately 2.9 million babies did not survive beyond the neonatal period (first 28 days of life) worldwide, ${ }^{5}$ and most of these deaths occurred in low/middle-income countries (LMICs). The leading causes of neonatal death globally were birth asphyxia, ${ }^{6}$ prematurity, sepsis and congenital malformation, ${ }^{7}$ indicating that interventions should focus on the healthcare management of the pregnancy, delivery and the postnatal periods. The WHO has made strong recommendations ${ }^{89}$ to: (1) at least four times of antenatal visits and at least three times of postpartum visits for the pregnant women during pregnancy; (2) delivery in hospital; (3) neonatal care. It had been estimated that if these programmes could be implemented worldwide, they may potentially reduce up to 1 million neonatal death per year. ${ }^{10}$ Therefore, effective interventions to promote neonatal healthcare would be important for LMICs, in reducing 
child mortality and achieving MDGs-4. Moreover, it has been found that home visits for neonatal care by community medical staff were associated with reduced neonatal mortality in resource-limited settings with poor healthcare accessibility. ${ }^{11}$ In $\mathrm{LMICs}^{7}$ like Brazil, Uganda and Ghana, the neonatal mortality had been largely reduced through better coverage of primary healthcare, hospital delivery and vaccinations.

The Chinese government endorsed the MDGs- 4 in $2000,{ }^{12}$ and had since introduced many programmes to improve maternal and neonatal healthcare, such as the 'Reducing Maternal Mortality and Eliminating Neonatal Tetanus' programme in $2000^{13}$ and the New Cooperative Medical Scheme in 2003. ${ }^{14}$ The under-5 mortality rate had reduced to 18.5 per 1000 live births by the end of $2008,{ }^{15}$ which not only made China achieve the MDGs- 4 years ahead of the 2015 target date, but also making China the eighth country to achieve this target worldwide. ${ }^{16}$ During this period, there was also a huge reduction in neonatal mortality rate (NMR) (33.1-10.2 per 1000 live births from 1991 to 2008). However, the gap of NMRs between urban and rural areas still exists. The NMR in urban and rural areas in 2008 were 5.0 and 12.3 per 1000 live births, respectively. ${ }^{15}$ This gap might cause by the regional inequality and insufficiency in health service delivery. ${ }^{17}$ In such context, the Chinese government launched the Basic Public Health Service (BPHS) project in 2009, ${ }^{18}$ which aimed at improving health service continuity and regional equality in the primary healthcare setting. This government-fund project originally included 9 categories in $2009^{19}$ and in 2019 it contained 17 categories. The services related to neonatal health included the maternal health management during pregnancy as well as the neonatal visits, which aimed at protecting the newborns from the beginning of the pregnancy to the early days of their births. To address the rural-urban disparities in neonatal health services, the BPHS project required standardised quality and quantity of health services during the implementation, and all the medical staff involved were required to be trained according to national standards.

By the year of 2017, the national NMR had decreased to 4.5 per 1000 live births (urban areas: 2.6; rural areas: 5.3) and the gap of NMR between urban and rural areas was narrowed. Nevertheless, whether this decrease in neonatal mortality and the improvement in rural-urban equality can be linked to the BPHS project has not been evaluated using robust methods, such as the segmented linear regression model. This model can quantify the level change and trend change while controlling for autocorrelated errors $^{2021}$ and adjusting for potential serial correlation of the data. It has been used in many previous studies to evaluate the effectiveness of health service programmes and medical interventions. ${ }^{22} 23$

This paper aims at assessing the effects of the BPHS project on reducing NMR in China. Specifically, trends of the annual NMR, the gap of NMR between urban and rural areas, maternal health management rate (MMR) and neonatal visit rate before and after the implementation of BPHS project would be measured. The associations between MMR, neonatal visit rate and NMR were also explored. The results of this study may provide references to health administrators in designing and implementing health projects aiming at reducing NMR and increasing regional equality in China and other LMICs.

\section{METHODS}

\section{Study design and data collection}

We conducted a longitudinal study of national NMR and the gap of NMR between urban and rural areas from 1991 to 2017, and neonatal related health service rate in BPHS project, including MMR and neonatal visit rate from 2000 to 2017 in China with the time-series design of beforeand-after assessment.

The neonatal related health services in BPHS project included the maternal health management during pregnancy and neonatal visit. The maternal health management include: the establishment of health record of mother and children within the first 13 weeks of pregnancy, the five antenatal visits within the first 13 weeks, 16-20 weeks, 21-24 weeks, 28-36 weeks, 37-40 weeks of pregnancy, respectively and postnatal visits within 7 days after delivery. Every antenatal examination included an inspection of family history, disease history, gynaecological examinations, blood routines, urine routines, liver function tests, kidney function tests, hepatitis B examination and so on. The medical staff also offer lifestyle, psychological and nutritional guidance for the mother. The neonatal visit refers to a visit to a newborn's home by medical staff within a week of discharge from the hospital, and the services include: enquiry the birth status (birth weight, body length), vaccination status (BCG (Bacillus Calmette-Guerin) vaccine and the first dose of hepatitis B vaccine), physical examination, feeding, sleeping, urination, jaundice, umbilical cord, temperature and oral cavity development of the neonatal; the guidance on feeding, development, disease prevention, injury prevention and oral healthcare are provided for parents according to the specific situation of the neonatal. For the neonatal with high risk factors such as low birth weight, premature birth, twins or birth defects, the number of neonatal visits should be increased according to the actual situation.

\section{Data sources}

The national NMR, urban NMR and rural NMR data were extracted from National Neonatal Mortality Surveillance System which was set up by the Chinese government in 1989. The sampling unit of the system was at the county (district) level. A total of 336 surveillance spots (126 urban areas and 210 rural areas), covering 31 provinces, were selected to record the changes in NMR. The data were collected by trained officials and verified by government administrators. In our study, the national NMR data and the gap of NMR between urban and rural areas between 1991 and 2017 were extracted and calculated from the system. The data of the neonatal related 
health services indicators in BPHS project from 2000 to 2017 were extracted from National Health Statistics Yearbook. The two indexes are the MMR and the neonatal visit rate. The data of these indicators were collected and verified at the provincial level and being uploaded to the Ministry of Health annually. The data are then presented in the National Health Statistic Yearbook published by the Ministry of Health. ${ }^{24}$

\section{Outcome measures}

The official definition of NMR of WHO is the deaths among the live births during the 28 completed days of life, which can be subdivided into early neonatal death (deaths between 0 and 7 completed days of birth) and late neonatal death (deaths after 7 days to 28 completed days of birth). ${ }^{25}$ According to the data collection protocol in China, the NMR was defined as the neonatal death in total and did not distinguish early neonatal death and late neonatal death. The gap of NMR between urban and rural areas equals the rural NMR minus urban NMR. The MMR and the neonatal visit rate were two of the government mandated indicators to measure the service provision of the maternal and neonatal health services in China. The MMR is defined as the proportion of pregnant women who have accepted at least five antenatal visits during pregnancy, one postpartum visit and establish the health records by the professionals during the first 13 weeks of pregnancy among all the women resulting in live births. The neonatal visit rate is defined as the proportions of the neonatal who have accepted at least one neonatal visit by medical staff at home within a week of hospital discharge among all the live births.

\section{Statistical analysis}

We analysed the time series data using the segmented linear regression model with statistical software (SPSS, V.21.0) to access changes in levels and trends of national NMR, the gap of NMR between urban and rural areas, MMR and neonatal visit rate before and after the introduction of BPHS project. We regarded the year 2009 as the intervention time point for the implementation of BPHS project. Segmented linear regression divided the time series into pre-2009 and post-2009 segments. We then compared the changes in trends and levels of national NMR, the gap of NMR between urban and rural areas, MMR and neonatal visit rate before and after the introduction of BPHS project. Pearson correlation analysis was conducted to measure the correlation between NMR and MMR, as well as NMR and neonatal visit rate. The statistical significance level was set at alpha $=0.05$.

\section{Patient and public involvement}

Patients and the public were not involved. Participants were not involved in developing the research questions or planning the study.

\section{RESULTS}

The yearly national NMR, urban NMR and rural NMR from 1991 to 2017 was displayed in online supplementary

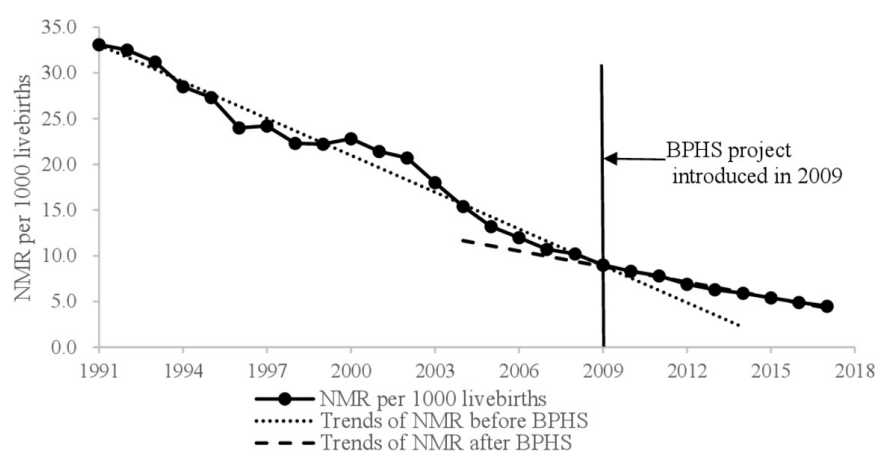

Figure 1 Level and trend changes of national NMR before and after the interventions. BPHS, Basic Public Health Service; NMR, neonatal mortality rate.

file 1. The national NMR ranged from 10.2 to 33.1 per 1000 live births from 1991 to 2008, which reduced to a range of 4.5-9.0 per 1000 live births during 2009-2017 after the introduction of BPHS project.

The NMR in urban and rural areas ranged between $5.0-12.5$ per 1000 live births and 12.3-37.9 per 1000 live births, respectively during 1991-2008, which went down to a range of 2.6-4.5 per 1000 live births and 5.3-12.3 per 1000 live births during 2009-2017. The gap of NMR between urban and rural areas ranged from 7.3 to 25.4 during 1991-2008, which reduced to 5.3-10.8 during 2009-2017 after the introduction of BPHS project.

MMR and neonatal visit rate also showed different trends before and after 2009 (online supplementary file 2). The MMR and neonatal visit rate ranged between 65.6-78.1 and 81.4-86.7, respectively before the introduction of BPHS project, and went up to a range of 80.9-91.6 and 87.1-93.9 after the intervention.

As presented in table 1 and figures 1-4, the segmented regression results showed that the trend of the yearly national MMR was -0.57 (95\% CI: -0.85 to $-0.28, \mathrm{p}<0.01$ ) after the interventions of BPHS project in 2009, which indicate a yearly decline of 0.57 neonatal death per 1000 live births. The gap of NMR between urban and rural areas show the decreasing trend after the introduction of BPHS project, with a decline of 0.49 annually, which indicate the

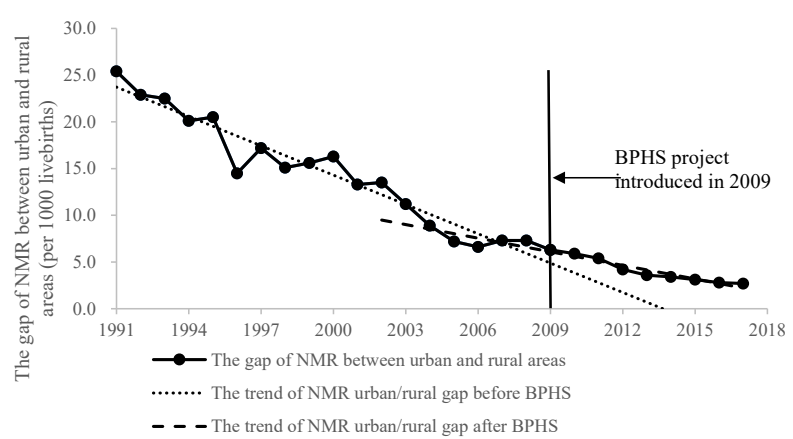

Figure 2 Level and trend changes of the gap of NMR between urban and rural areas before and after the intervention. BPHS, Basic Public Health Service; NMR, neonatal mortality rate. 


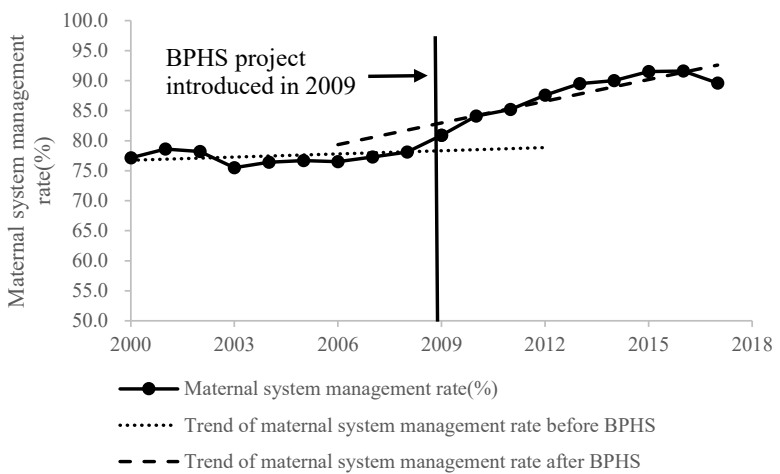

Figure 3 Level and trend changes of maternal health management rate before and after the interventions. BPHS, Basic Public Health Service.

gap of NMR between urban and rural areas continuous to narrow. For the MMR and neonatal visit rate, the trend slowly increased before the introduction of BPHS project in 2009. There was an immediate increase in MMR (4.75, $95 \%$ CI: 1.86 to $7.63, \mathrm{p}<0.01)$ and neonatal visit rate $(2.88$, $95 \%$ CI: 1.14 to $4.61, \mathrm{p}<0.01$ ), respectively when the interventions were introduced (table 1 and figures 1-4). The trend of all the rates showed rapid increases of postintervention, with all results being statistically significant.

The Pearson correlation analysis results indicated a strong negative correlation between national NMR and MMRs as well as neonatal visit rates. The correlation between NMR and MMRs was $-0.79(\mathrm{p}<0.01)$, and the correlation between NMR and neonatal visit rate was $-0.76(\mathrm{p}<0.01)$.

\section{DISCUSSION}

This study observed the continuous decreasing trend of the national NMR and the gap of NMR between urban and rural areas before and after the introduction of BPHS project, while the neonatal related service volume kept a decreasing or slowly increasing trend from 2000 to 2008,

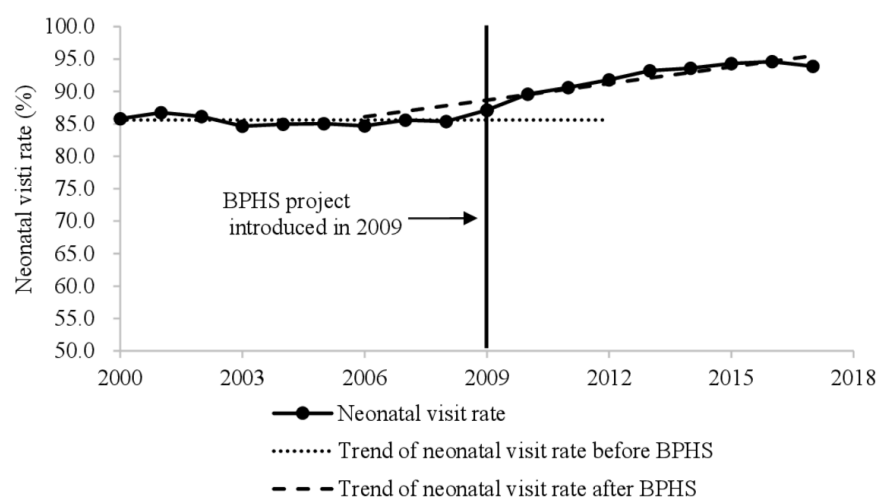

Figure 4 Level and trend changes of neonatal visit rate before and after the interventions. BPHS, Basic Public Health Service. and the trends changed into rapid increasing from 2009 to 2017 after the introduction of BPHS project.

These results indicated that the national NMR was further decreased and the neonatal related services were increased with the implementation of the BPHS project, the inequality and disparities between urban and rural areas were also diminishing. The effectiveness of BPHS project confirmed the importance of project design and government commitment in improving neonatal health services and reducing NMR. ${ }^{26} 27$

The BPHS project had a national coverage and abundant government funding compared with previous programmes. The neonatal related health services in BPHS project covered almost all the key stages from foetal formation to neonatal. ${ }^{28}{ }^{29}$ Maternal health management was designed to start from early pregnancy to postpartum period, in order to fully monitor the growth and development of the foetus, and reducing the incidence of low birth weight and birth defects. The neonatal visit helped to ensure the health status of the newborns through physical examination and health education to the parents. The BPHS project was designed to achieve the full cycle of health monitoring and healthcare for newborns from pregnancy to neonatal, which could be key to its effectiveness. The evidences in India, ${ }^{28} \mathrm{Brazil}^{30}$ and many LMICs showed that they had achieved significant decrease in NMR through maternal and neonatal health services, at least three times of antenatal care and postpartum care $^{31}$ were provided during the whole pregnancy, which confirmed the potential effect of maternal and neonatal health services in reducing NMR.

The segmented linear regression analysis showed the effectiveness of BPHS project on improving neonatal related health services. There were statistically significant immediate effects in improving MMRs and neonatal visit rates. Statistically significant long-term effects on these neonatal related services were also shown, since it kept a decreasing trend before the introduction of the project. It implied that the neonatal related health services of the BPHS project was not a one-time campaign but rather resulted in long-term benefit, essential for the continuous improving on neonatal health services.

The regression results of national NMR and the gap of NMR between urban and rural areas showed both significant descending trend before and after the introduction of BPHS project, but the descending trends were slower compared with the trends before the introduction of BPHS project. The slower decreasing trend may be due to the boundary effect. It had been reported that the national NMR is more likely to be affected by the rural NMR, ${ }^{32} 33$ which could be also explained in Chinese condition. The NMR in rural areas presented a more rapid decreasing trend from 1991 to 2008 which decreased from 37.9 to 12.3 per 1000 live births and then decreased from 10.8 to 5.3 per 1000 live births from 2009 to 2017. It is difficult to keep the same rapid decreasing trend when the NMR has already been reduced to a relatively low level. Compared with urban areas, where it took 14 years to reduce NMR 
Table 1 Estimated level and trend changes of national NMR, the gap of NMR between urban and rural areas, maternal health management rate and neonatal visit rate before and after the interventions outcome variables

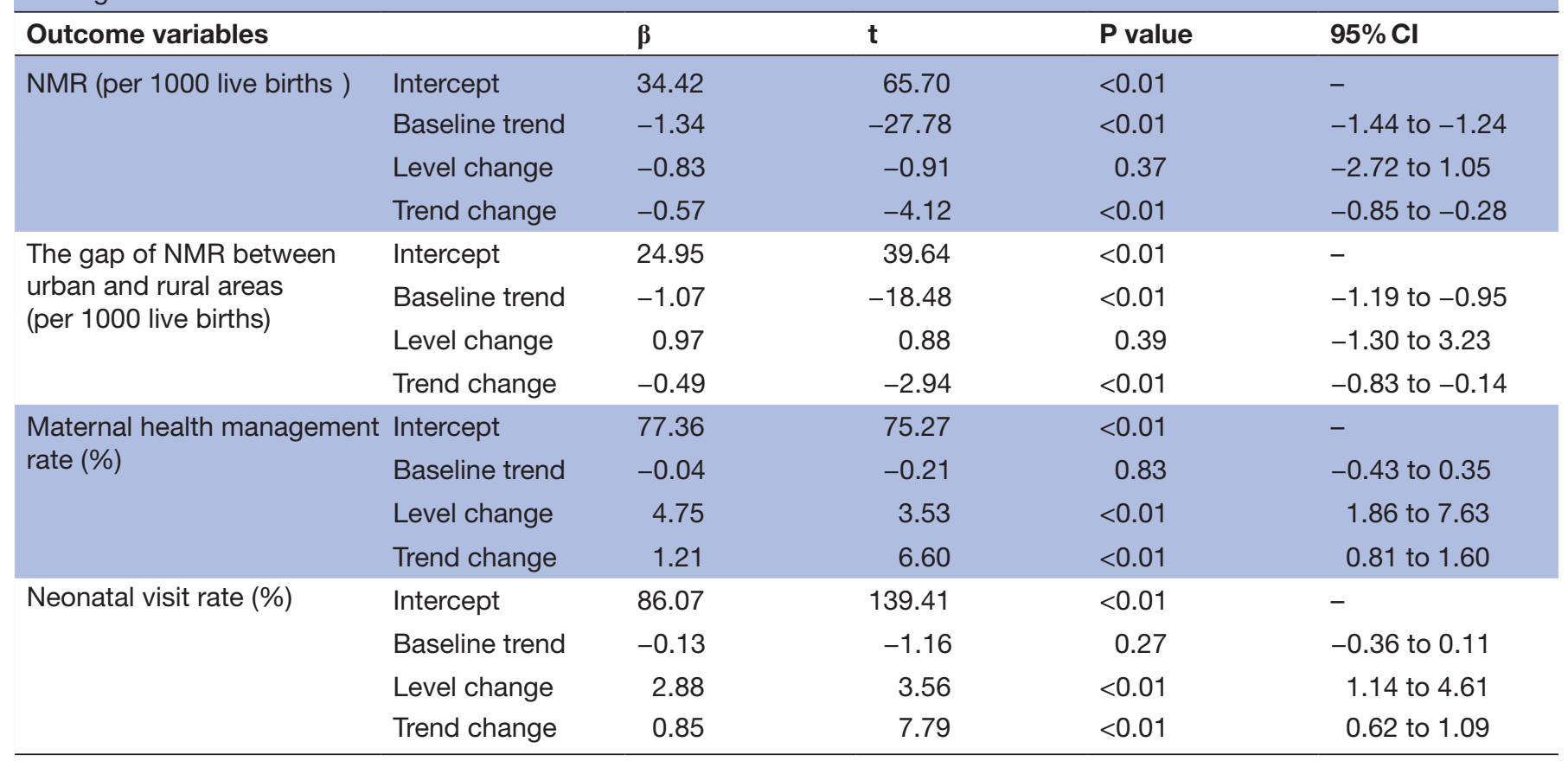

NMR, neonatal mortality rate.

from 10.6 to 5.0 per 1000 live births from 1995 to 2008 , it took only 9 years for rural areas to reduce NMR from 10.8 to 5.3 per 1000 live births from 2009 to 2017 after the introduction of BPHS project. Meanwhile, the ratio of rural to urban NMR narrowed from 3.0 to 2.5 from 1991 to 2008 after 17 years and the ratio had decreased from 2.4 to 2.0 after 9 years' implementation of BPHS project from 2009 to 2017. These findings provided evidence in supporting of the effectiveness of BPHS project on improving neonatal health services, equality of health services and NMR reduction.

China had achieved the target to further reduce NMR and to narrow the gap of NMR between urban and rural areas partly through the implementation of the BPHS project. However, many LMICs, especially African countries still have low levels of neonatal health services and high levels of NMR. The BPHS project in China provided an example for other LMICs on how to carry out continuous, high-quality neonatal health services with the support of relevant evidence. First, the central and provincial government need to provide subsidies for the community health centres to cover the cost of maternal health management and neonatal visit. This approach could reduce the financial pressures on primary medical institutions. Second, the project should be designed to cover all the maternal and neonatal populations, regardless of their socioeconomic status or urban/ rural household and so on. This approach increased the equality and coverage of the neonatal healthcare services. Third, the neonatal services should be provided by trained medical staffs and should be preventative in nature. Health examinations can help identifying higher risk newborns and facilitate timely interventions. This approach reflects the combination of population strategy and high-risk strategy.

Our study has several limitations. First, the data regarding the causes of the neonatal death were not available, which limited our ability to analyse the risk factors of the neonatal mortality and advise future policies on NMR reduction. Furthermore, due to limited data accessibility, this study did not assess the effectiveness of the BPHS project at the provincial or county levels, nor did it control for other factors affecting the neonatal health outcomes, such as neonatal care quality, ${ }^{34}$ delivery place ${ }^{35}$ and so on.

\section{CONCLUSION}

The BPHS project, a national project funded by the Chinese government, played an important role in improving neonatal health services, further reducing NMR and diminishing inequality in healthcare between urban and rural areas. This case study provides evidence for other LMICs to implement organisational and structural changes in reducing NMR and improve neonatal care.

Future studies could focus on assessing the effectiveness of the BPHS project at provincial or county levels in order to understand to mechanism behind the NMR reduction. The fact that the decreasing rate of NMR was slower after the project introduction in 2009 also needs further monitoring and exploration.

Acknowledgements We are grateful for the professors and lecturers at the Chinese Academy of Medical Science and Peking Union Medical College for their 
instructive suggestions and helpful comments. We also appreciate the government officials in the Ministry of Health for their efforts in collecting, verifying and reporting the data used in this paper.

Contributors PZ: study design, database establishment, statistical analysis and paper writing. XH: study design and manuscript revision. YZ: data collection, database establishment. LYou and LYang: study design and data collection. YL: study design, comment on statistical analysis and modification of the paper.

Funding This work was supported by National Health Commission of the People's Republic of China (no 2018-24).

Competing interests None declared.

Patient consent for publication Not required.

Provenance and peer review Not commissioned; externally peer reviewed.

Data availability statement All data relevant to the study are included in the article or uploaded as supplementary information. Access to National Health Statistic Yearbook data from 2000 to 2017 and National Neonatal Mortality Surveillance System data from 1991 to 2017 are managed and provided by National Health Commission of the People's Republic of China following an online address (http://www.nhc.gov.cn/).

Open access This is an open access article distributed in accordance with the Creative Commons Attribution Non Commercial (CC BY-NC 4.0) license, which permits others to distribute, remix, adapt, build upon this work non-commercially, and license their derivative works on different terms, provided the original work is properly cited, appropriate credit is given, any changes made indicated, and the use is non-commercial. See: http://creativecommons.org/licenses/by-nc/4.0/.

ORCID iD

Yuanli Liu http://orcid.org/0000-0002-5559-1149

\section{REFERENCES}

1 GBD 2016 Mortality Collaborators. Global, regional, and national under-5 mortality, adult mortality, age-specific mortality, and life expectancy, 1970-2016: a systematic analysis for the global burden of disease study 2016. Lancet 2017;390:1084-150.

2 Countdown to 2030 Collaboration. Countdown to 2030: tracking progress towards universal coverage for reproductive, maternal, newborn, and child health. Lancet 2018;391:1538-48.

3 Silver KL, Singer PA. SDGs: start with maternal, newborn, and child health cluster. Lancet 2014;384:1093-4.

4 Liu L, Oza S, Hogan D, et al. Global, regional, and national causes of child mortality in 2000-13, with projections to inform post-2015 priorities: an updated systematic analysis. Lancet 2015;385:430-40.

5 Lawn JE, Blencowe H, Oza S, et al. Every newborn: progress, priorities, and potential beyond survival. Lancet 2014;384:189-205.

6 de Almeida MFB, Moreira LMO, Vaz dos Santos RM, et al. Early neonatal deaths with perinatal asphyxia in very low birth weight Brazilian infants. J Perinatol 2015;35:954-7.

7 Lehtonen L, Gimeno A, Parra-Llorca A, et al. Early neonatal death: a challenge worldwide. Semin Fetal Neonatal Med 2017;22:153-60.

8 BP VJ. WHO antenatal care randomized trial: manual for the implementation of the new model. Geneva: WHO, 2002.

9 WHO. WHO recommendations on postnatal care of the mother and newborn. Geneva: WHO, 2013.

10 Little G, Niermeyer S, Singhal N, et al. Neonatal resuscitation: a global challenge. Pediatrics 2010;126:e1259-60.

11 Das JK, Rizvi A, Bhatti Z, et al. State of neonatal health care in eight countries of the SAARC region, South Asia: how can we make a difference? Paediatr Int Child Health 2015;35:174-86.

$12 \mathrm{He} \mathrm{C}$, Liu L, Chu Y, et al. National and subnational all-cause and cause-specific child mortality in China, 1996-2015: a systematic analysis with implications for the sustainable development goals. Lancet Glob Health 2017;5:e186-97.

13 Liang J, Li X, Kang C, et al. Maternal mortality ratios in 2852 Chinese counties, 1996-2015, and achievement of millennium development goal 5 in China: a subnational analysis of the global burden of disease study 2016. Lancet 2019;393:241-52.

$14 \mathrm{Wu}$ J, Deaton S, Jiao B, et al. The cost-effectiveness analysis of the new rural cooperative medical scheme in China. PLoS One 2018;13:e0208297.

15 Ministry of Health of the People's Republic of China. China health statistics yearbook. [In Chinese]. Beijing: Ministry of Health of the People's Republic of China, 2009.

16 Wang Y, Li X, Zhou M, et al. Under-5 mortality in 2851 Chinese counties, 1996-2012: a subnational assessment of achieving MDG 4 goals in China. Lancet 2016;387:273-83.

17 Song P, Theodoratou E, Li X, et al. Causes of death in children younger than five years in China in 2015: an updated analysis. $J$ Glob Health 2016;6:020802.

18 Zhao Y, Huo Zhen'ang, Wu J, et al. Impact on the performance of health workers adopted performance-related contracts in the provision of basic public health service at village and township levels. Iran J Public Health 2013;42:358-67.

19 Ministry of Health of the People's Republic of China. National standard of Basic Public Health Service (2009)[In Chinese]. Beijing: Ministry of Health of the People's Republic of China, 2009.

20 Wagner AK, Soumerai SB, Zhang F, et al. Segmented regression analysis of interrupted time series studies in medication use research. J Clin Pharm Ther 2002;27:299-309.

21 Lagarde M, do Hto. How to do (or not to do). Assessing the impact of a policy change with routine longitudinal data. Health Policy Plan 2012;27:76-83.

22 Agboola S, Simons M, Golas S, et al. Health care cost analyses for exploring cost savings opportunities in older patients: longitudinal retrospective study. JMIR Aging 2018;1:e10254.

23 Hurtado-Navarro I, García-Sempere A, Rodríguez-Bernal C, et al. Impact of drug safety warnings and Cost-Sharing policies on osteoporosis drug utilization in Spain: a major reduction but with the persistence of over and underuse. data from the ESOSVAL cohort from 2009 to 2015. Front Pharmacol 2019;10:768.

24 Fang J, Kaufman J. Reproductive health in China: improve the means to the end. Lancet 2008;372:1619-20.

25 WHO. Trends in maternal mortality: 1990 to 2015. Geneva: WHO, 2015.

26 Shiffman J, Sultana S. Generating political priority for neonatal mortality reduction in Bangladesh. Am J Public Health 2013;103:623-31.

27 Smith SL, Shiffman J, Kazembe A. Generating political priority for newborn survival in three low-income countries. Glob Public Health 2014:9:538-54

28 Sankar MJ, Neogi SB, Sharma J, et al. State of newborn health in India. J Perinatol 2016;36:S3-8.

29 Yonemoto N, Dowswell T, Nagai S, et al. Schedules for home visits in the early postpartum period. Cochrane Database Syst Rev 2017;8:Cd009326.

30 Guerra AB, Guerra LM, Probst LF, et al. Can the primary health care model affect the determinants of neonatal, post-neonatal and maternal mortality? A study from Brazil. BMC Health Serv Res 2019;19:133.

31 Singh PK, Rai RK, Alagarajan M, et al. Determinants of maternity care services utilization among married adolescents in rural India. PLoS One 2012;7:e31666.

32 Khajavi A, Pishgar F, Dehghani M, et al. Socioeconomic inequalities in neonatal and postneonatal mortality: evidence from rural Iran, 1998-2013. Int J Equity Health 2017;16:83.

33 Akinyemi JO, Bamgboye EA, Ayeni O. Trends in neonatal mortality in Nigeria and effects of bio-demographic and maternal characteristics. BMC Pediatr 2015:15:36.

34 Gogia S, Sachdev HPS. Home-Based neonatal care by community health workers for preventing mortality in neonates in low- and middle-income countries: a systematic review. J Perinatol 2016;36 Suppl 1:S55-73.

35 Debelew GT, Afework MF, Yalew AW. Determinants and causes of neonatal mortality in Jimma Zone, Southwest Ethiopia: a multilevel analysis of prospective follow up study. PLoS One 2014;9:e107184 